\title{
MdNup62 interactions with MdHSFs involved in flowering and heat-stress tolerance in apple
}

\author{
Chenguang Zhang ${ }^{1}$, Na An ${ }^{2}$, Peng Jia ${ }^{1}$, Wei Zhang ${ }^{1}$, Jiayan Liang ${ }^{1}$, Hua Zhou ${ }^{1}$, Zhang \\ dong $^{3}$, Juanjuan $\mathrm{Ma}^{1}$, zhao caiping ${ }^{3}$, Mingyu $\mathrm{Han}^{1}$, xiaolin $\mathrm{ren}^{4}$, and libo xing ${ }^{5}$ \\ ${ }^{1}$ Northwest A\&F University \\ ${ }^{2}$ College of Horticulture, Northwest A\&F University \\ ${ }^{3}$ Northwest A \& F University \\ ${ }^{4}$ Northwest Agriculture and Forestry University \\ ${ }^{5}$ Affiliation not available
}

January 17, 2021

\begin{abstract}
Because of global warming, the apple flowering period is occurring significantly earlier, increasing the probability and degree of freezing injury. Moreover, extreme hot weather has also seriously affected the development of apple industry. Nuclear pore complexes (NPCs) are main channels controlling nucleocytoplasmic transport, but their roles in regulating plant development and stress responses are still unknown. Here, we analysed the components of the apple NPC and found that MdNup62 interacts with MdNup54, forming the central NPC channel. MdNup62 was localized to the nuclear pore, and its expression was significantly up-regulated in 'Nagafu No. 2' tissue-cultured seedlings subjected to heat treatments. To determine MdNup62's function, we obtained MdNup62-overexpressed (OE) Arabidopsis and tomato lines that showed significantly reduced hightemperature resistance. Additionally, OE-MdNup62 Arabidopsis lines showed significantly earlier flowering compared with wildtype. Furthermore, we identified 62 putative MdNup62-interacting proteins and confirmed MdNup62 interactions with multiple MdHSFs. The OE-MdHSFA1d and OE-MdHSFA9b Arabidopsis lines also showed significantly earlier flowering phenotypes than wild-type, but had enhanced high-temperature resistance levels. Thus, MdNUP62 interacts with multiple MdHSFs during nucleocytoplasmic transport to regulate flowering and heat resistance in apple. The data provide a new theoretical reference for managing the impact of global warming on the apple industry.
\end{abstract}

MdNup62 interactions with MdHSFs involved in flowering and heat-stress tolerance in apple

Chenguang Zhang+, Na An+, Pen Jia+, Wei Zhang, Jiayan Liang, Hua Zhou, Dong Zhang, Juanjuan Ma, Caiping Zhao, Mingyu Han, Xiaolin Ren, Libo Xing*

${ }^{1}$ College of Horticulture, Northwest A\&F University, 712100 Yangling, Shaanxi, P. R. China

\section{${ }^{+}$Equal contributors}

*Corresponding author:

Libo Xing

E-mail: libo_xing@nwsuaf.edu.cn ;

Tel.: +8615129227289;

ORCID: https://orcid.org/0000-0002-8918-7128; 
Address: 3 Taicheng Road, Yangling 712100, Shaanxi, P. R. Chin

Run title: MdNup62 interactions with MdHSFs regulate flowering and heat-stress tolerance

\begin{abstract}
Because of global warming, the apple flowering period is occurring significantly earlier, increasing the probability and degree of freezing injury. Moreover, extreme hot weather has also seriously affected the development of apple industry. Nuclear pore complexes (NPCs) are main channels controlling nucleocytoplasmic transport, but their roles in regulating plant development and stress responses are still unknown. Here, we analysed the components of the apple NPC and found that MdNup62 interacts with MdNup54, forming the central NPC channel. MdNup62 was localized to the nuclear pore, and its expression was significantly upregulated in 'Nagafu No. 2' tissue-cultured seedlings subjected to heat treatments. To determineMdNup62 's function, we obtained MdNup62 -overexpressed (OE) Arabidopsis and tomato lines that showed significantly reduced high-temperature resistance. Additionally, OE-MdNup62 Arabidopsis lines showed significantly earlier flowering compared with wild-type. Furthermore, we identified 62 putative MdNup62 -interacting proteins and confirmed MdNup62 interactions with multipleMdHSFs. The OE-MdHSFA1d and OE-MdHSFA9b Arabidopsis lines also showed significantly earlier flowering phenotypes than wild-type, but had enhanced high-temperature resistance levels. Thus, MdNUP62 interacts with multiple MdHSFs during nucleocytoplasmic transport to regulate flowering and heat resistance in apple. The data provide a new theoretical reference for managing the impact of global warming on the apple industry.
\end{abstract}

\title{
Keywords
}

Apple, Flowering, heat stress, nuclear pore complex,MdNup62 , MdHSFs

\section{Introduction}

Apple (Malus $\times$ domestica Borkh.) is a widely cultivated and economically important fruit crop in temperate regions worldwide owing to its high nutritional value, good storage, and lengthy supply period. Fuji apple is the main cultivar in China, but there are cultivation and production problems, including flowering difficulties and severe alternate bearing (Fan, Zhang, Lei, Chen, Xing, Ma, Zhao \& Han, 2016, Guitton, Kelner, Velasco, Gardiner, Chagné \& Costes, 2012). However, with global warming, an increase in the average temperature in winter will result in earlier apple flowering (Romanovskaja \& Bakšiene, 2009, 刘璐, 郭梁, 李曼华, 傅玮东 \& 栾青, 2020), and if there is cold weather in early spring, then significant flower and fruit losses will result. Additionally, at present, extreme hot weather occurs frequently in summer, causing other problems, such as growth impairment and production decline (Yao, Song, Wang, Song, Jiao, Wang \& Zheng, 2020, ZHOU, SUN, LIU, JIN, ZHANG \& WEI, 2016), which have seriously affected the development of the apple industry in China.

Floral induction pathways have been extensively studied, and six signalling pathways, photoperiodic, vernalization, autonomic, gibberellin, temperature-sensitive, and age, regulate flowering in the model plant Arabidopsis thaliana (Bäurle \& Dean, 2006, Komeda, 2004, Teotia \& Tang, 2015). In apple, the functions of some key flowering-related genes have been well studied in recent years, such as APETALA1 (AP1), LEAFY (LFY), FLOWERING LOCUS T (FT), and TERMINAL FLOWER 1 (TFL1 ). For instance, overexpression of MdMADS5 , a putative homolog of $A P 1$, leads to significant early flowering in Arabidopsis (Kotoda, Wada, Kusaba, Kano-Murakami, Masuda \& Soejima, 2002). AFL1 and AFL2, two orthologues of LFY , have been isolated from apple buds, and their overexpression lines in Arabidopsis flower significantly earlier than wild type (WT), with the overexpression of $A F L 2$ leading to a more obvious early-flowering phenotype than the overexpression of AFL1 (Wada, Cao, Kotoda, Soejima \& Masuda, 2002). Apple anti-TERMINAL FLOWER 1 transgenic lines flower significantly earlier than the WT, with the earliest flowering at 8 months, while the WT did not flower for 6 years (Kotoda, Iwanami, Takahashi \& Abe, 2006). The overexpression of the apple FT gene in Arabidopsis, poplar, and apple results in significantly earlier flowering in these plants, and transgenic poplar and apple flower during in vitro cultivation (Trankner, Lehmann, Hoenicka, Hanke, Fladung, Lenhardt, Dunemann, Gau, Schlangen, Malnoy \& Flachowsky, 2010). The overexpression of 
MdFT1 and MdFT2 independently in Arabidopsis results in significantly earlier flowering under both longand short-day conditions ( $\mathrm{Li}$, Tao, Yao, Hao \& You, 2010). Through transcriptome analyses, the induction of apple flower buds was found to be regulated by sugar and hormone signalling pathways (Xing, Zhang, Li, Shen, Zhao, Ma, An \& Han, 2015). Other omics studies have revealed the molecular mechanisms involved in responses to exogenous treatments, such as sugar (Liu, Feng, Pan, Zhong, Chen, Yuan \& Li, 2016), 6-benzylaminopurine (Li, Zhang, An, Fan, Zuo, Zhang, Zhang, Gao, Han \& Xing, 2019), and gibberellins (Zhang, Gottschalk \& van Nocker, 2019), and their effects on the flowering of apples. However, research on apple flowering is still relatively limited.

A nuclear pore complex (NPC) is composed of a class of nucleoporins (Nups ) located in the nuclear pore (Tamura, Fukao, Iwamoto, Haraguchi \& Hara-Nishimura, 2010). More than 30 Nups have been identified in Arabidopsis and 38 members have been identified in apple (Tamuraet al., 2010, Zhang, An, Jia, Zhang, Liang, Zhang, Zhou, Ma, Han, Xing \& Ren, 2020). Some Nups interact and form three subcomplexes: Nup62 , Nup93 , and Nup107-160 (Tamura et al. , 2010, Zhu, Wang, Tang, Hsu, Xie, Du H, Yang, Tao \& Zhu, 2017).Nups control the transport of substances, such as RNA and proteins, between the nucleus and cytoplasm (Parry, 2013, Zhang, Wang, Kim, Yan, Yan, Pang \& Hua, 2020). Proteins rely on importin $\alpha$, importin $\beta$, and Ran-GTP complexes to pass through the NPC and enter the nucleus (Gorlich, Seewald \& Ribbeck, 2003, Hill, 2009, Takizawa, Weis \& Morgan, 1999). Importin $\beta 1$ interacts with importin $\alpha$, Ran-GTP, and Nup62 directly in Arabidopsis, which further illustrates that plants and humans share a similar nuclear transport mechanism (Luo, Wang, Ji, Fang, Wang, Tian \& Li, 2013). Nups play important roles in regulating plant growth and development, as well as biotic and abiotic stresses (Parry, 2013, Xu \& Meier, 2008, Yang, Wang, Chu, Zhu \& Zhang, 2017). For example, HOS1 , Nup96 , Nup54 ,Nup58 , Nup62 , Nup136 , and Nup160 are important for plant flowering (Cheng, Zhang, Huang, Huang, Zhu, Chen, Miao, Liu, Fu \& Wang, 2020, Jung, Park, Lee, To, Kim, Seki \& Park, 2013, Lazaro, Mouriz, Piñeiro \& Jarillo, 2015, Parry, 2014, Tamuraet al. , 2010). HOS1 , Nup85 , Nup96 , andNup133 participate in abiotic stress pathways (Dong, Agarwal, Zhang, Xie \& Zhu, 2006, Dong, Hu, Tang, Zheng, Kim, Lee \& Zhu, 2006, Ishitani, Xiong, Lee, Stevenson \& Zhu, 1998, Zhang et al., 2020, Zhu et al. , 2017). MOS7, Nup96 , Nup160, andSec1 play important roles in plant immunity (Cheng, Germain, Wiermer, Bi, Xu, García, Wirthmueller, Després, Parker, Zhang \& Li, 2009, Roth \& Wiermer, 2012, Zhang \& Li, 2005), and Nup96 ,Nup160 , and TPR affect hormone signalling pathways (Jacob, Mongkolsiriwatana, Veley, Kim \& Michaels, 2007, Parry, Ward, Cernac, Dharmasiri \& Estelle, 2006, Robles, Deslauriers, Alvarez \& Larsen, 2012, Wiermer, Cheng, Imkampe, Li, Wang, Lipka \& Li, 2012, Xu, Rose, Muthuswamy, Jeong, Venkatakrishnan, Zhao \& Meier, 2007).

Heat shock factors (HSFs) are important components of signal transduction and play important roles in diverse stress pathways (Scharfa, Berberich, Ebersberger \& Nover, 2012). The HSF family in plants has more members (21 HSFs in Arabidopsis) and more complex regulatory mechanisms (Nover, Bharti, Doring, Mishra, Ganguli \& Scharf, 2001, Wang, Liu, Yu, Guo, Chen, Jiang, Xu, Fang, Wang, Zhang \& Chen, 2020) than in vertebrates (4 HSFs ) or Drosophila (only $1 H S F$ ). The structures of plant HSFs are relatively consistent from the N- to C-termini. The N-terminus has a DNA-binding domain, followed by heptad hydrophobic repeats involved in oligomerization, and a nuclear localization signal. The C-terminus contains a nuclear export signal and short peptide motifs. HSFs may be divided into three classes, A, B, and C, on the basis of their structural differences (Nover et al. , 2001). Class A has the C-terminal short peptide AHA domain, which has an activator function, while the B and C classes lack this domain (Kotak, Port, Ganguli, Bicker \& von KoskullDöring, 2004). HSFs specifically identify and bind heat shock elements (HSEs), which contain nGAAnnTTCn or nTTCnnGAAn in the downstream target genes' promoters (Littlefield \& Nelson, 1999). Class A members (HSFA1a , HSFA1b ,HSFA1d , HSFA1e , HSFA2 , and HSFA3 ) positively regulate plant heat tolerance (Charng, Liu, Liu, Chi, Wang, Chang \& Wang, 2007, Nishizawa-Yokoi, Nosaka, Hayashi, Tainaka, Maruta, Tamoi, Ikeda, Ohme-Takagi, Yoshimura, Yabuta \& Shigeoka, 2011, Qian, Chen, Liu, Yang, Li \& Zhang, 2014, Schramm, Larkindale, Kiehlmann, Ganguli, Englich, Vierling \& Von Koskull-Döring, 2008, Tian, Wang, Zhao, Lan, Yu, Zhang, Qin, Hu, Yao, Ni, Sun, Rossi, Peng \& Xin, 2020), while, in contrast, Class B HSFs (HSFB1 and HSFB2b ) negatively regulate heat-induced HSFs and plant heat tolerance (Ikeda, Mitsuda \& Ohme-Takagi, 2011). In addition to responding to heat stress, plant HSFs also participate in other stress 
pathways. For instance, HSFA1a -overexpression (OE) plants have enhanced tolerance under low/high $\mathrm{pH}$ levels and to hydrogen peroxide treatments (Qian et al. , 2014). HsfA2 knockout (KO) mutants (hsfa2) and double mutants (hsfa1a/hsfa1b) both lose their heat-dependent adaptability to hypoxia (Banti, Mafessoni, Loreti, Alpi \& Perata, 2010). Under high-light stress, the photosystem II activity of KO-hsfa1d/a1e mutants decreases, while that of WT plants remains high (Nishizawa-Yokoi et al. , 2011). The drought and salt tolerance levels of Oryza sativa OE- $H S F B b$ transgenic lines significantly decrease, but they are significantly enhanced in $O s H S F B b$-RNAi lines (Xiang, Ran, Zou, Zhou, Liu, Zhang, Peng, Tang, Luo \& Chen, 2013). Apple HSFA 8 a regulates the synthesis of flavonoids, thus enhancing the drought tolerance of apple (Wang et al. , 2020). In addition, some HSFs (HSFA2 , HSFA1E , and HSFA 4C ) appear to be involved in plant flowering pathways (Chen, Zhao, Ge, Han, Ning, Luo, Wang, Liu, Zhang \& Wang, 2018, Liu, Feng, Gu, Deng, Qiu, Li, Zhang, Wang, Deng, Wang, He, Bäurle, Li, Cao \& He, 2019). Thus, HSFs may play important roles in plant development and stress tolerance.

Currently, there are no reported functional studies of Nups in apple. Nup62 is a member of the Nup62 subcomplex in the central core of the nuclear pore (Tamura et al., 2010, Zhang et al., 2020), and nup62 A. thaliana mutants have been reported to flower early, indicating Nup62 's involvement in flowering pathways (Parry, 2014). In this study, we characterized appleNup62, which showed a high transcription level at the flower bud developmental stage and was responed to high temperature. The overexpression of MdNup62 in Arabidopsis resulted in earlier flowering compared with WT. Moreover, The overexpression of MdNup62 in Arabidopsis and tomato both reduced heat resistance. Further, we performed a yeast two-hybrid $(\mathrm{Y} 2 \mathrm{H})$ sieve library experiment to screen for proteins that interact with MdNup62, and the interactions between MdNup62 and the MdHSFs were confirmed. And the overexpression of MdHSFA1d and MdHSFA9bindependently in Arabidopsis resulted in earlier flowering and enhancing heat resistance. Thus, MdNup62 and the MdHSFs regulate flowering and respond to temperature changes. These results provide a theoretical reference for managing the impact of global warming on the apple industry.

\section{Materials and methods}

\section{Plant materials and growth conditions}

The plant materials were 6-year-old apple trees ('Fuji' /T337/Malus robusta Rehd.) growing in the experimental orchard of the Horticulture College of Northwest A \& F University (108 $04^{\prime}$ E, 34deg16' N). We collected new shoots $(2-3 \mathrm{~mm}$ in diameter) near the tips, fully expanded leaves near buds, flower buds, blooming flowers, and young fruit, which were immediately frozen in liquid nitrogen and stored at -80degC for later use.

The 'Fuji' plants were grown on MS medium containing $0.1 \mathrm{mg}^{*} \mathrm{~L}^{-1}$ indolebutyric acid and $0.6 \mathrm{mg}^{*} \mathrm{~L}^{-1} 6$ benzylaminopurine under long-day conditions (16h-light/8h-dark) at $24 \mathrm{deg} \mathrm{C}$ and were subcultured every 45 days. Arabidopsis plants were grown under long-day conditions (16h-light/8h-dark) at 22degC. Tomato plants ('Ailsa Craig') were grown under long-day conditions (16h-light/8h-dark) at 25degC.

\section{Heat map, protein alignment, and phylogenetic analysis}

Based on RNA-seq data of our laboratory, the heat map of apple different tissues was constructed using $\mathrm{MeV}$ (Multiple Experiment Viewer) software. A protein sequence alignment of Nup62 from six Rosaceae plants was performed using DNAMAN software. The Nup62 protein sequences were obtained from the GDR database (https://www.rosaceae.org/). The phylogenetic tree was constructed using MEGA-X software.

\section{RNA extraction and qRT-PCR analysis}

Total RNA was extracted from apple trees, Arabidopsis seedlings, tomato seedlings, and apple seedlings using an RNA Plant Plus Reagent Kit (TIANGEN, Beijing, China). The RNA was used as the template to synthesize cDNA with a PrimeScript RT Reagent Kit (Takara, Shiga, Japan). The qRT-PCR analysis was conducted on a StepOnePlus Real-Time PCR System (Thermo Fisher Scientific, USA). The reaction solution contained $10 \mu \mathrm{L}$ SYBR Green I Master Mix (CWBIO, Beijing, China), $0.5 \mu \mathrm{mol} \cdot \mathrm{L}^{-1}$ primers (SANGON BIOTECH, Shanghai, China), and $1 \mu \mathrm{L}$ each template in a total volume of $20 \mu \mathrm{L}$. The PCR program was 
as follows: $95^{\circ} \mathrm{C}$ for $3 \mathrm{~min} ; 40$ cycles of $94^{\circ} \mathrm{C}$ for $15 \mathrm{~s}, 60^{\circ} \mathrm{C}$ for $20 \mathrm{~s}$, and $72^{\circ} \mathrm{C}$ for $15 \mathrm{~s}$. All the samples were analysed with three biological replicates, each comprising three technical replicates. Relative gene expression levels were calculated in accordance with the $2^{-\mathrm{Ct}}$ method (Livak \& Schmittgen, 2001). The primers used for qRT-PCR (Table S4)

were synthesized by the Sangon Biotechnology Co. Ltd. (Shanghai, China).

\section{Subcellular localization}

The open reading frames (ORFs) of the MdNup62 , MdHSFA1d, and MdHSFA9b genes were inserted independently into the pCAMBIA2300-EGFP vector to generate the 35S::MdNup62 -EGFP, 35S::MdHSFA1d -EGFP, and 35S::MdHSFA9b -EGFP recombinant plasmids, respectively. These recombinant plasmids were inserted independently into Agrobacterium tumefaciens strain GV3101 cells. The GV3101 cells containing these recombinant plasmids were then infiltrated into tobacco leaves. GV3101 cells containing the pCAMBIA2300-EGFP vector (35S::EGFP) served as the control. After an additional 3 days of growth in the dark, green fluorescent protein (GFP) signals in transformed tobacco leaves were detected using a Leica TCS SP8 SR Laser Scanning Confocal Microscope (Leica, Germany). The primers used are listed in Table S5.

\section{Genetic transformation}

The genetic transformations were performed in accordance with published methods for Arabidopsis (Clough \& Bent, 1998) and tomato ('Ailsa Craig') (Liu, Sun, Liu, Shi, Chen \& Zhao, 2019) plants. The transgenic Arabidopsis and tomato lines were selected on MS plates supplemented with $50 \mathrm{mg} \cdot \mathrm{L}^{-1}$ and 100 $\mathrm{mg}^{*} \mathrm{~L}^{-1}$ kanamycin, respectively.

\section{Yeast two-hybrid (Y2H) assay}

The MdNup62 ${ }^{508-613}$ truncated sequence was cloned into the pGBKT7 vector to generate theMdNup62 508-613 _pGBKT7 recombinant plasmid. The MdHSFAs' ORFs were inserted individually into the pGADT7 vector to generate the MdHSFAs-pGADT7 recombinant plasmids. The recombinant plasmids were inserted into Gold Yeast Two-Hybrid cells, which were then grown on a selective medium. The primers used are listed in Table S5.

\section{Split luciferase (LUC) complementation}

The full-lengthMdHSFA1dand MdHSFA9b coding sequences were cloned independently into the CLUC vector, while MdNup62 was cloned into the NLUC vector. The split-LUC complementation assay was performed with tobacco leaves. The reconstituted LUC activity was detected in the dark using a Princeton Lumazone Pylon 2048B cooling camera (Princeton, USA). The LUC activity was quantified using the Dual-Luciferase Reporter Assay System (Promega, USA). The primers used are listed in Table S5.

\section{Pull-down assays}

The ORFs of MdNup62 and MdHSFA9b were cloned into the pET-28a and pGEX-6p-1 vectors, respectively, and subsequently overexpressed independently in Escherichia coli BL21(DE3) (Transgene). The pull-down assays were conducted using the His-Tagged Protein Purification Kit (Clontech) in accordance with the manufacturer's instructions. The primers used are listed in Table S5.

\section{Heat-tolerance assays}

The 'Fuji' plants at 30 days after propagation were used for the $45 \mathrm{deg} \mathrm{C}$ heat treatment. We collected leaf samples before and at 1,3 , and $6 \mathrm{~h}$ after the treatment. The samples were immediately frozen in liquid nitrogen and stored at $-80 \operatorname{deg} \mathrm{C}$ for later use.

Two-week-old transgenic Arabidopsis and 3-week-old transgenic tomato were used for the heat treatment in an artificial climate chamber. OE-MdNup62 A. thaliana lines were subjected to $45 \operatorname{deg} \mathrm{C}$ for $12 \mathrm{~h}$, and 
OE-MdHSFA9b and OE-MdHSFA1d A. thalianalines were subjected to 45degC for $16 \mathrm{~h}$. OE-MdNup62 tomato lines were subjected to $45 \operatorname{deg} \mathrm{C}$ for $14 \mathrm{~h}$.

\section{Evaluation of stress tolerance}

The superoxide dismutase, peroxidase, and catalase activities and the malondialdehyde and $\mathrm{H}_{2} \mathrm{O}_{2}$ levels were detected using the corresponding Suzhou Comin Biotechnology test kits (Suzhou Comin Biotechnology Co., Ltd, Suzhou, China). The presence of $\mathrm{O}^{2-}$ in leaf samples was determined by staining with nitro blue tetrazolium.

\section{Statistical analyses}

Statistical analyses were performed using SPSS software. Data are reported as means +- SDs. Asterisks $(*)$ indicate significant differences between treatments as assessed by Student's t-test at $\mathrm{P}<0.05\left(^{*}\right)$ and $\mathrm{P}<$ $\left.0.01{ }^{* *}\right)$. Different lowercase letters above the bars indicate significant differences $(\mathrm{P}<0.05$, Tukey's test).

\section{Results}

\section{Apple NPC structure and composition, and its expression patterns}

Compared with vertebrate, apple NPC consists of 38 NUP proteins, but missing some Nups, such as Nup153, Nup358, Pom121, etc. Refer to the structure of vertebrate NPC (Tamura et al. , 2010), we devided apple NPC into five parts: Cytoplasmic filaments (Nup214 and Nup88), Cytoplasmic and Nuclear ring (Nup98, RAE1, and Nup107-160 Subcompex), Scaffold and central channal (GP210, NDC1, Nup62 Subcompex, and Nup93 Subcompex), Nuclear basket (Nup50 and Nup136), and Distal ring (Tpr/NUA), as well as GLE1, ALADIN, CG1, and HOS1 also participate in NPC constitution (Figure 1a). Additionally, MdNup62 interacts with MdNup54, forming the central apple NPC channel involving in nucleocytoplasmic transport (Figure S1) (Zhang et al. , 2020).

We examined the expression patterns of NPC components in different tissues of several apple varieties (Figure 1b; Table S1). The expression levels of MdNup62 as central channel component showed significantly higher in buds, stem, roots than in fruit of apples, but other channel component MdNup54 showed significantly low expression levels in all tissues compared with MdNup62, indicating that MdNup62 play a key role in regulation of growth and stress response by controlling nucleocytoplasmic transport in apple.

\section{Feature, expression, and subcellular localization analyses of $M d N u p 62$}

We initially performed a simple bioinformatics analysis ofMdNup62. A phylogenetic tree of Nup62 from six Rosaceae plants (Rosa chinensis, Pyrus communis ,Prunus persica, M. domestica, Rubus occidentalis, and Fragaria vesca) was constructed using MEGA-X. MdNup62 was most closely related to the Nup62 of pear (Figure 2a). The aligned protein sequences revealed a conserved Nsp1_C domain (Figure 2b). The subcellular localization of MdNup62 was determined by introducing 35S::MdNup62 -GFP into tobacco leaves (Figure 2c). Tobacco leaves transformed with the empty vector 35S::GFP were used as controls. In the tobacco leaves expressing 35S::MdNup62 -GFP, the GFP signal was observed only in the nuclear pore, while the GFP signal was detected throughout the control tobacco leaf cells, indicating thatMdNup62 localized to the nuclear pore.

The transcript levels of MdNup62 in different tissues were determined using qRT-PCR (Figure 2d). The highest expression level was in flower buds. An MdNup62 expression analysis during the flower bud developmental stages revealed that the expression level was stable at 30 to 60 days after flowering and reached its highest level at 70 days after flowering (Figure 2e). Thus, MdNup62 maintained a high expression level during flower bud induction, indicating that it may be related to bud differentiation in apple.

We exposed apple tissue-cultured seedlings to a heat treatment. The reactive oxygen species (ROS) accumulation in leaves increased from 0 to $6 \mathrm{~h}$ under heat-treatment conditions (Figure 2f). Moreover, the expression level of MdNup62 was determined at different times during the high-temperature treatment (Figure 2g). MdNup62 was significantly induced by high temperature, and its expression level was highest at 1 
$\mathrm{h}$ after exposure to the high temperature. Thus, MdNup62 may be involved in the heat-resistance pathway of apple.

\section{Overexpression of $M d N u p 62$ promotes flowering}

To confirm MdNup62 's role in flowering, we performed an Agrobacterium-mediated genetic transformation of MdNup62 intoA. thaliana. We found that OE-MdNup62 lines flowered significantly earlier than WT (Figure 3a). Additionally, OE-MdNup62 lines had significantly fewer rosette leaves than WT during bolting (Figure 3b). The presence of the transgene in OE-MdNup62 lines was confirmed using genomic PCR (Figure S2a), semi-quantitative RT-PCR (Figure 3c), and qRT-PCR (Figure 3d). The transcript levels of floweringrelated genes were analysed by qRT-PCR (Figure 3e). The expression levels of AtFT , AtLFY, and AtAP1 significantly increased in OE-MdNup62 lines compared with WT. This demonstrated that the overexpression of MdNup62promoted flowering in Arabidopsis.

\section{Overexpression of $M d N u p 62$ reduces high-temperature resistance}

Because MdNup62 was induced by high temperature, we investigated the high-temperature resistance function of MdNup62 . OE-MdNup62 Arabidopsis lines were subjected to a high-temperature (45degC) treatment (Figure 4a). Additionally, the survival rate of transgenic Arabidopsis was significantly lower than that of WT (Figure 4b). We also performed a qRT-PCR analysis of A. thaliana HSPs (AtHSP101, AtHSP22ER, AtHSP21.0 , andAtHSP70T-2 ) (Figure 4c). Their expression levels in transgenic Arabidopsis were reduced under high-temperature conditions. Consistently, after the heat treatment, the ROS accumulation in leaves was clear greater in OE-MdNup62 lines compared with WT (Figure 5a). In addition, the malondialdehyde and $\mathrm{H}_{2} \mathrm{O}_{2}$ levels were significantly greater than in WT (Figure 5b,c). Moreover, the superoxide dismutase, peroxidase, and catalase activities were lower in OE-MdNup62lines than in WT (Figure $5 \mathrm{~d}-\mathrm{f}$ ). High-temperature resistance assays were carried out in transgenic tomato plants (Figure 6a). As in transgenic $A$. thaliana, the survival rate of transgenic tomato was significantly reduced compared with WT (Figure 6b). The presence of the transgene in OE-MdNup62lines was confirmed by genomic PCR, and qRT-PCR (Figure 6c,d). The expression levels of HSPs (HSP101, HSP22-ER ,HSP21.0, and HSP70T-2 ) in transgenic tomato were significantly reduced under high-temperature conditions compared with under normal growth conditions (Figure 6e). These results indicate that MdNup62 reduces plant high-temperature resistance.

\section{MdNup62-interacting protein screening}

To further reveal the function of MdNup62 , we conducted a $\mathrm{Y} 2 \mathrm{H}$ sieve library experiment using a MdNup62 truncated body (MdNup62 $2^{508-613}-p G B K T r 7$ ) that is not self-activated. We identified 62 putative MdNup62 -interacting proteins (Table S3). Some transcription factors were identified, such as HSFs (MdHSFA1d , MdHSFA1e , MdHSFA9 , MdHSF30 ,MdHSF1 , and MdHSF8 ), as well as MdMYB21 ,MdMYC2 , $M d G A T A 11$, and MdBAK1. In addition, some enzymes and other functional genes were found. Because transcription factors that have transcriptional regulatory functions must be transported into the nucleus, and because MdNup62 has regulatory effects on the transport of the proteins, we hypothesized that MdNup62 interacts with these $M d H S F$ s and controls their transport.

\section{MdNup62 interacts with MdHSFs}

We cloned parts of the MdHSF s (MdHSFA1a/b/d/e andMdHSFA9a/b) independently into the pGADT7 vector and then cotransformed each with MdNUP62 ${ }^{508-613}-p G B K T 7$.MdNup62 interacted with these MdHSF s (Figure 7a).Additionally, we used MdHSFA9b in pull-down assays. The recombinant MdNup62$H I S$ fusion protein was purified withMdHSFA9b-GST, but not with GST alone (Figure 7b). The splitLUC complementation assay revealed that the co-expression of MdNup62-NLUC with MdHSFA1d -CLUC or MdHSFA9b-CLUC resulted in a higher LUC activity than the other combinations (Figure 7c-e). These results confirmed the interaction between MdNup62and both MdHSFA1D and MdHSFA9b.

Feature, expression, and subcellular localization analyses of MdHSFA $9 b$ and MdHSFA1d 
Phylogenetic tree analysis showed that Apple and Arabidopsis HSFs were divided into four groups(I,II,III,IV), with $M d H S F A 1 a / b / d / e$ in groupII, and MdHSFA $9 a / b$ in groupI(Figure 8a). We also examined the expression patterns of MdHSFs in different tissues of several apple varieties (Figure 8b; Table S2). And the expression levels of MdHSFA1a/b/d/e

and MdHSFA9a/b showed significantly higher in buds.

The subcellular localizations of MdHSFA9b and MdHSFA1dwere studied by independently introducing 35S::MdHSFA9b -EGFP and 35S::MdHSFA1d-EGFP, respectively, into tobacco leaves (Figure 8c). Tobacco leaves transformed with the empty vector 35S::EGFP served as controls. In the tobacco leaves expressing 35S::MdHSFA9b -EGFP and 35S::MdHSFA1d -EGFP, the GFP signals were observed in both the nucleus and cytoplasm, while the GFP signal was detected throughout the control tobacco leaf cells, indicating that MdHSFA9b andMdHSFA1d localized to both the nucleus and cytoplasm. The co-localization of MdNup62 with both MdHSFA9b andMdHSFA1d further verified their interactions.

A tissue-specific expression analysis revealed that MdHSFA1d was expressed highest in flower buds and stems. The highest expression level of MdHSFA9b was in stems, but the expression levels in the other tissues were also high. Subsequently, the expression levels of MdHSFA9b and MdHSFA1d remained high during the flower bud developmental stages, while the highest was at 70 days after flowering (Figure 8d). These results indicated that MdHSFA9b and MdHSFA1d maintained high expression levels during flower bud induction, suggesting that they may be involved in the bud differentiation of apple.

\section{Overexpression of $M d H S F A 9 b$ and $M d H S F A 1 d$ promotes flowering}

To verify the flowering phenotype of HSFs, we performed Agrobacterium-mediated genetic transformations of MdHSFA9b andMdHSFA1d into A. thaliana . Like OE-MdNup62, OE-MdHSFA9b and OE-MdHSFA1d lines flowered significantly earlier than WT (Figures 9a and S3a). Additionally, they also had significantly fewer rosette leaves than WT during bolting (Figures $9 \mathrm{~b}$ and S3b). We also performed genomic PCR (Figure S2b, c), semi-quantitative RT-PCR (Figures 9c and S3c), and qRT-PCR (Figures 9d and S3d) to confirm the presence of the transgene in the OE-MdHSFA9b and OE-MdHSFA1d lines. The transcript levels of AtFT , $A t L F Y$, and AtSOC1 were significantly increased in OE-MdHSFA9b and OE-MdHSFA1d lines compared with WT (Figures 9e and S3e).

\section{Overexpression of MdHSFA $9 b$ and MdHSFA1d enhances high-temperature resistance}

To study the high-temperature resistance phenotypes of MdHSFA9band MdHSFA1d, we also exposed OEMdHSFA9b and OE-MdHSFA1d transgenic plants, respectively, to high-temperature (45degC) conditions (Figures 10a and S4a). The survival rates of OE-MdHSFA9b and OE-MdHSFA1d lines were significantly greater than that of WT (Figures 10b and S4b). Consistently, the ROS accumulation in leaves decreased in transgenic plants after the high-temperature treatment (Figure S5a, b). We also performed a qRT-PCR analysis of A. thaliana HSPs (AtHSP101,AtHSP22-ER, AtHSP21.0 , and AtHSP70T-2 ) (Figures 10c and S4c), and their expression levels in transgenic $A$. thalianaincreased under high-temperature conditions compared with under normal growth conditions. These results indicated that MdHSFA9b andMdHSFA1d enhance plant high-temperature resistance.

\section{Discussion}

Plant flowering has always been an important topic in crop and horticultural sciences, and issues with apple flowering have long hindered the development of the apple industry in China (Fan et al. , 2016, Guitton et al. , 2012). The Nups control protein transport between the nucleus and cytoplasm, and they participate in a variety of biological processes, including flowering (Parry, 2013, Zhang et al. , 2020).HOS1 regulates the binding of some nuclear genes toFLOWERING LOCUS C (FLC) chromatin at low temperatures and weakens the transcriptional inhibition of FLC by HDA6 (Junget al. , 2013). In A. thaliana, Nup96 promotes the stability of HOS1, and HOS1 conjugates and degrades $C O$, then promotes $F L C$ expression, leading to delayed flowering. In addition, HOS1 increases the stability of Nup 96 and thus maintains this regulatory pathway to control the flowering time (Cheng et al. , 2020, Lazaro et al. , 2015). Mutations in Nup54, 
Nup58, Nup62 , Nup136, and Nup160 have resulted in a prominent earlier flowering phenotype compared with WT (Parry, 2014, Tamura et al. , 2010). In the present study, MdNup62 maintained a high expression level during flower development. To verify the flowering function of MdNup62, we determined the flowering phenotypes of OE-MdNup62 A. thaliana lines. Interestingly, the phenotypes of the overexpression lines were consistent with Arabidopsis deletion mutants and showed obvious early flowering. Previous studies found that both Nup62 deletion mutants and overexpression strains of Arabidopsis have increased the sensitivities to auxin, indicating that the overexpression does not result in a functional gain, but rather a functional loss, like the mutant (Boeglin, Fuglsang, Luu, Sentenac, Gaillard \& Cherel, 2016). Therefore, the overexpression of MdNup62 in this study may also result in a functional loss. However, MdNup62 is involved in the flowering pathway.

With global warming, extreme high-temperatures will occur more frequently, which will seriously affect the normal growth and development of plants (Yao et al. , 2020, ZHOU et al. , 2016). And Nups are involved in temperature-stress responses. HOS1 is an important negative regulator of cold-signal transduction in plant cells, and hos1-1 has poor cold resistance compared with WT (Ishitaniet al. , 1998). HOS1 specifically binds to and degrades $I C E 1$, thereby reducing cold resistance (Dong et al. , 2006). The expression levels of cold-resistance-related genes, such as $C B F$, in atnup160 mutants were impaired and seedlings grew slowly, especially at low temperatures (Dong et al. , 2006). Nup85 and Nup133 control mRNA output only under warm conditions and are more sensitive to transcription factor localization at warm temperatures (Zhang et al. , 2020). In this study, MdNup62 responded to high-temperature stress in apple. However, OEMdNup62 lines had reduced high-temperature resistance in both Arabidopsis and tomato. By analysing the relative expression levels of HSPs (HSP101, HSP22-ER , HSP21.0 , and HSP70T-2 ) in transgenic plants, we found no obvious correlations between OE-MdNup62 lines and WT at a normal growth temperature, but OE-MdNup62 lines had significantly lower HSP expression levels than WT under high-temperature conditions.

In plants, Nup-interacting proteins have been studied (Cheng et al. , 2020, Zhang et al. , 2020, Zhu et al. , 2017), and some potential Nup85 -interacting proteins have been identified by immunoprecipitation and subsequent mass spectrometry in Arabidopsis, such as the Nup107-160 subcomplex (Nup160, Nup133 ,Nup43 , Nup96 , Nup107, Seh1, andSec13), several mediator subunits (MED16, MED14, and MED18 ), HOS1, and Sec13A. The interactions between Nup85 and three proteins, HOS1, Sec13A, andMED18 , have been confirmed (Zhu et al. , 2017). Additionally, a direct interaction between Nup96 and HOS1 in Arabidopsis has also been reported (Cheng et al. , 2020). In our previous study, the interaction between MdNup54 andMdNup62 was confirmed in apple (Zhang et al. , 2020). However, there are no reports of direct interactions between transcription factors and Nups in plants. We previously identified an interaction between apple MdNup54 and MdKNAT4/6 using a yeast double-hybridization test, but further verification is needed (Zhang et al. , 2020). In this study, we verified direct interactions between MdNup62 and MdHSF s, indicating that the Nups may directly recognize related transcription factors and thus regulate their transport. This provides a new direction of study for Nups.

Because of the early flowering of OE-MdNup62 Arabidopsis lines, MdHSF s that interact with MdNup62 may be also involved in the flowering pathway. Consistent with this conjecture, some HSFs are associated with flowering (Chenet al. , 2018, Liu et al. , 2019). HSFA1E and HSFA4C directly target and positively regulate the flowering geneSOC1 in lettuce (Chen et al. , 2018). Arabidopsis HSFA2 directly targets and promotes the expression of $R E F 6$, and the REF6-HSFA2 loop directly targets and activates HTT5, which coordinates early flowering (Liu et al. , 2019). In this study, we found that MdHSFA9b and MdHSFA1d maintained high expression levels during flower bud induction. Additionally, OE-MdHSFA9b and OE-MdHSFA1dArabidopsis lines flower significantly earlier than WT. This suggests that MdHSFA9b and MdHSFA1d promote plant flowering.MdNup62, MdHSFA9b, and MdHSFA1d share the same flowering phenotype, possibly because the overexpression of MdNup62 fosters HSF accumulation in the nucleus, promoting the expression of downstream flowering-related genes and advancing flowering.

HSFs play important roles in regulating plant resistance to high temperatures. HSFA1 positively regulates 
the heat tolerance of tomato, the expression of HSFA2 is dependent on HsfA1, and the thermotolerance of the posttranscriptional silencing of the HsfA1 gene in protoplasts can be restored by plasmid-borneHsfA2 (Mishra, 2002). In Arabidopsis, HSFA3 directly up-regulates the expression of Hsp18.1-Cl and Hsp26.5$M I I$, and both HSFA3 mutants and RNAi significantly reduce high temperature resistance (Schramm et al. , 2008). The overexpression of HsfA1a increases Hsp18.2 and Hsp 70 expression levels, as well as heatshock tolerance (Qian et al. , 2014).HSFA1b regulates high-temperature resistance through OPR3and the jasmonate signalling pathway (Tian et al. , 2020).HSFA1d and HSFA1e activate HsfA2 transcription, and a double knockout of HSFA1d and HSFA1e impairs tolerance to heat-shock stress (Nishizawa-Yokoi et al. , 2011). In Arabidopsis, HsfA2 acts as a heat-induced transactivator to maintain the expression levels of HSPs and prolong the duration of acquired thermotolerance (Charng et al. , 2007). InMedicago truncatula , HSFA9 plays important roles in thermotolerance (Zinsmeister, Berriri, Basso, Ly Vu, Dang, Lalanne, Da Silva, Leprince \& Buitink, 2020). In the current study, we obtained similar results for MdHSFA9b and MdHSFA1d. The expression levels of HSPs in the two overexpression Arabidopsis lines were significantly greater than in WT, and both lines had enhanced high-temperature resistance levels. Like the flowering and auxin phenotypes (Boeglin et al. , 2016), the opposite phenotypes between OE-MdNup62 and OEMdHSFA9b, OE-MdHSFA1dindicates that the overexpression of MdNup62 may also result in a lack of function under heat-stress conditions. Similar to the results of this study, Zhang et al. (2020) found that nup85 and nup133 increase the ubiquitous protoplast (nucleus and cytosol) signals of IAA17 and PIF4 at $28 \mathrm{degC}$ compared with at $22 \mathrm{degC}$. Furthermore, the nup 96 and hos1 mutants show significant increases in the ubiquitous localizations of IAA17 andPIF 4 signals at 28degC ( $72 \%$ and $66 \%$, respectively) compared with 22degC (40\% and 49\%, respectively)(Zhang et al. , 2020, 北京爱琴海乐之技术有限公司, 2005)(北京爱 琴海乐之技术有限公司, 2005; Zhang et al. , 2020)(Zhang et al. , 2020). Thus, the nuclear accumulations of the IAA17 and PIF4 proteins innup85, nup96, nup133, and hos1 are reduced compared with WT, and the defects are more severe at $28^{\circ} \mathrm{C}$. Therefore, we hypothesized that the transport of MdHSFA9b, MdHSFA1d, and other MdHSF s is inhibited in OE-MdNup62 lines at high temperatures, resulting in the inhibition of the transcription of downstream HSP s, which further reduces high-temperature resistance.

On the basis of these findings, we constructed a hypothetical model of MdNup62-related pathways involved in high-temperature resistance (Figure 11). At normal temperature, apple MdHSFs were not induced, and not much transported into nucleus that cannot lead to up-regulate expression of MdHSPs in WT and OEMdNup62. However, at high temperature, apple MdHSFs were significantly induced, and then transported into the nucleus through NPC channels to promote the expression of MdHSPs in WT, in which enhanced high-temperature resistance. But for OE-MdNup62 lines, the structure of the apple NPC changed, and blocked the transport of high temperature induced MdHSFs into the nucleus that cannot induce much MdHSPs expression causing heat injuring (Figure 11). Additionally, OE-MdNup62, OE-MdHSFA9b and OE-MdHSFA1d lines showed significant early flowering phenotype compared with WT (Figure 3, 9; Figure S3).

In conclusion, temperature is an important factor affecting flowering. With global warming, apple flowering will occur earlier, increasing the risk of chilling-related injury. Moreover, extreme hot weather is also occurring frequently. Both climatic conditions seriously affect the development of the apple industry. MdNup62 interacts withMdHSFs to regulate flowering and heat-resistance pathways in plants. Thus, both MdNup62 and the MdHSFs regulate flowering and respond to temperature changes. This research provides a theoretical reference for managing the impact of global warming on the apple industry.

\section{Author contributions}

Libo Xing and Chenguang Zhang conceived and designed the experiment. Chenguang Zhang, Peng Jia, Na An, Wei Zhang, Jiayan Liang, Hua Zhou performed the experiment. Chenguang Zhang and Peng Jia analyzed the data. Chenguang Zhang and Libo Xing wrote the manuscript. 


\section{Acknowledgments}

This work was financially supported by the National Natural Science Foundation of China (31801813; 32072522); the China Postdoctoral Science Foundation (2018M631207, 2017M623254); Natural Science Foundation of Shaanxi Province (2020JQ-248).

\section{Conflict of interest statement}

The authors declare that the research was conducted in the absence of any commercial or financial relationships that could be construed as a potential conflict of interest.

\section{Figure legends}

Figure 1. The nuclear pore complex (NPC) structure and composition in Vertebrate and Malus.

(a) A schematic of the nuclear pore with the cytoplasmic side at the top and the nuclear basket at the bottom for Vertebrate (left) and Malus (right).

(b) Tissue specific expression patterns of apple NPC components by RNA-seq. The full names of the different abbreviations are as follows, 'Nagafu No.2' long branches flower buds (FLB), 'Nagafu No.2' short branches flower buds (FSB), 'Nagafu No.2' axillary buds (FYB), 'Qinguan' axillary buds (QYB), 'Nagafu No.2' fruit (FR), 'Yanfu No.3' stem tip (YF3J), 'Yanfu No.6' stem tip (YF6J), and 'M9-T337' root (T337R). Each number after the abbreviation represents a biological repetition.

Figure 2. Identification and analysis of MdNup62.

(a) Phylogenetic analysis of Rosaceae Nup62 . (b) The conservative domain of Rosaceae Nup62 . (c) Subcellular localization of MdNup62 . The upper panel shows 35S::EGFP, and the lower panel shows 35S::MdNup62EGFP. (d) and (e) Analyses of MdNup62 expression levels in diverse 'Nagafu No. 2' apple tissues (d) and in different flower bud developmental stages of 'Nagafu No. 2' (e). (f) The phenotype of 'Nagafu No. 2' tissuecultured seedlings (upper panels) and the in situ accumulation of superoxide radical $\left(\mathrm{O}^{2-}\right)$ at $0,1,3$, and 6 $\mathrm{h}$ under heat treatment conditions (lower panels). Bar $=1 \mathrm{~cm}$. (g)MdNup62 expression levels in 'Nagafu No. 2' tissue-cultured seedling leaves at $0,1,3$, and $6 \mathrm{~h}$ under heat-treatment conditions. Each sample was analysed with three biological replicates, each comprising three technical replicates. Means followed by different lowercase letters are significantly different at the 0.05 level.

Figure 3. MdNUP62 promotes flowering in Arabidopsis.

(a) Phenotype of the MdNUP62 -overexpression Arabidopsis line for flowering time. Bar $=2 \mathrm{~cm}$. (b) Statistical analysis of rosette leaves of Arabidopsis thaliana during bolting. Asterisks denote significant differences as determined by a t-test $\left({ }^{*} \mathrm{P}<0.05\right)$. (c) Semi-quantitative RT-PCR analysis of MdNup62 expression in Arabidopsis samples. (d) qRT-PCR analysis of MdNup62 expression in Arabidopsis samples. Asterisks denote significant differences as determined by a t-test $\left({ }^{*} \mathrm{P}<0.01\right)$. (e) Relative expression levels of flowering genes (AtFT, AtLFY, AtSOC1, andAtAP1 ) in WT and MdNup62 -overexpression lines. Each sample was analysed with three biological replicates, each comprising three technical replicates. Asterisks denote significant differences as determined by a t-test $\left({ }^{*} \mathrm{P}<0.05\right)$.

Figure 4. MdNup62reduced high-temperature resistance in Arabidopsis.

(a) Phenotype of the MdNup62 -overexpression Arabidopsis line for high-temperature resistance. Bar = $1 \mathrm{~cm}$. (b) Survival rates of WT and MdNup62 -overexpression Arabidopsis lines after the high-temperature treatment. Asterisks denote significant differences as determined by a t-test $(* * \mathrm{P}<0.01)$. (c) Relative expression levels of high-temperature resistance-related genes (AtHSP101,AtHSP22.0-ER, AtHSP21, and 
AtHSP70T-2 ) in WT andMdNup62 -overexpression lines at the normal (22degC) temperature and $1 \mathrm{~h}$ after exposure to the high-temperature (37degC) treatment. Each sample was analysed with three biological replicates, each comprising three technical replicates. Asterisks denote significant differences as determined by a t-test $(* * \mathrm{P}<0.01)$.

Figure 5. Changes in the level of accumulated ROS and activities of ROS-scavenging enzymes in OE-MdNup62 and WT Arabidopsis leaves under heat-stress conditions.

(a) In situ accumulations of superoxide radicals $\left(\mathrm{O}^{2-}\right)$ before (upper panels) and after (lower panels) heat treatment as revealed by nitro blue tetrazolium staining. (b) and (c) Quantitative measurement of $\mathrm{H}_{2} \mathrm{O}_{2}$ and malondialdehyde concentrations in Arabidopsis leaves treated with and without the high temperature. (d)-(f) Activities of superoxide dismutase (SOD), peroxidase (POD), and catalase (CAT) at $6 \mathrm{~h}$ after the heat treatment. Each sample was analysed with three biological replicates, each comprising three technical replicates. Means followed by different lowercase letters are significantly different at the 0.05 level.

Figure 6. MdNup62 reduced high-temperature resistance in tomato.

(a) Phenotype of the MdNup62 -overexpression tomato line for high-temperature resistance. Bar $=5 \mathrm{~cm}$. (b) Survival rates of WT and MdNup62 -overexpression tomato lines after the high-temperature treatment. Asterisks denote significant differences as determined by a t-test $(* * \mathrm{P}<0.01)$. (c) qRT-PCR analysis of MdNup62expression levels in tomato samples. Asterisks denote significant differences as determined by a t-test (**P < 0.01). (d) Genomic PCR analysis of MdNup62 transgenic tomato lines. (e) Relative expression levels of high-temperature resistance-related genes (SlHSP101, SlHSP22.0-ER, SlHSP21, andSlHSP70T-2 ) in WT and MdNup62 -overexpression lines at the normal temperature (22degC) and $1 \mathrm{~h}$ after exposure to the high-temperature $(45 \mathrm{degC})$ treatment. Each sample was analysed with three biological replicates, each comprising three technical replicates. Asterisks denote significant differences as determined by a t-test $\left({ }^{*} \mathrm{P}\right.$ $<0.05)$.

Figure 7. MdNup62 interacts with MdHSFs.

(a) Interactions between $M d N u p 62^{508-613}$ and MdHSF s (MdHSFA9a/b and MdHSFA1a/b/d/e ) in Y2H assays. The MdNup62 $2^{508-613}$ truncated sequence was cloned into pGBKT7, whereas MdHSFA s (MdHSFA9a/b and $M d H S F A 1 D a / b / d / e$ ) were cloned independently into the pGADT7 vector. Empty pGADT7 plus MdNup62 $2^{508-613}$-pGBKT7 was used as the control. (b) Interactions between MdNup62 andMdHSFA9b in the pull-down assay. Western blotting with a GST antibody revealed that MdNup62-HIS was pulled down byMdHSFA9b-GST . (c)-(e) Interactions between MdNup62 and both MdHSFA9b and MdHSFA1d in a luciferase (LUC) complementation experiment. Empty NLUC and empty CLUC,MdNup62 -NLUC plus empty CLUC, empty NLUC plus MdHSFA9b, and MdHSFA1d -CLUC were used as controls. The LUC complementation experiment was repeated three times, with consistent results. Asterisks denote significant differences as determined by t-tests $(* * \mathrm{P}<0.01)$.

Figure 8. Subcellular localization and expression analyses of MdHSFA9b and MdHSFA1d.

(a) Phylogenetic analysis of HSFs in Malus and Arabidopsis.(b) Tissue specific expression patterns of apple MdHSFs by RNA-seq. The full names of the different abbreviations are as follows, 'Nagafu No.2' long branches flower buds (FLB), 'Nagafu No.2' short branches flower buds (FSB), 'Nagafu No.2' axillary buds (FYB), 'Qinguan' axillary buds (QYB), 'Nagafu No.2' fruit (FR), 'Yanfu No.3' stem tip (YF3J), 'Yanfu No.6' stem tip (YF6J), and 'M9-T337' root (T337R). Each number after the abbreviation represents a biological repetition. (c) Subcellular localizations of MdHSFA9b and MdHSFA1d. The upper panel shows 35S::EGFP, the middle panel shows 35S::MdHSFA9b -EGFP, and the lower panel shows 35S::MdHSFA1d -EGFP. (d) Analyses of MdHSFA9b andMdHSFA1d expression levels in diverse apple 'Nagafu No. 2' tissues and in different flower bud developmental stages of 'Nagafu No. 2'. Each sample was analysed with three biological replicates, each comprising three technical replicates. Means followed by different lowercase letters are significantly different at the 0.05 level.

Figure 9. MdHSFA9b promotes flowering in Arabidopsis. 
(a) Phenotype of the MdHSFA9b -overexpression Arabidopsis line for flowering time. Bar $=2 \mathrm{~cm}$. (b) Statistical analysis of rosette leaves of Arabidopsis thaliana during bolting. Asterisks denote significant differences as determined by a t-test $\left({ }^{*} \mathrm{P}<0.05\right)$. (c) Semi-quantitative RT-PCR analysis of MdHSFA9b expression in Arabidopsis samples. (d) qRT-PCR analysis of MdHSFA9b expression in Arabidopsis samples. Asterisks denote significant differences as determined by a t-test $\left({ }^{*} \mathrm{P}<0.01\right)$. (e) Relative expression levels of flowering genes (AtFT, AtLFY, AtSOC1, and AtAP1 ) in WT and MdHSFA9b -overexpression lines. Each sample was analysed with three biological replicates, each comprising three technical replicates. Asterisks denote significant differences as determined by a t-test $\left({ }^{*} \mathrm{P}<0.05\right)$.

Figure 10. MdHSFA9b enhanced high-temperature resistance in Arabidopsis.

(a) Phenotype of the MdHSFA9b -overexpression Arabidopsis line for high-temperature resistance. Bar $=2$ $\mathrm{cm}$. (b) Survival rates of WT and MdHSFA9b -overexpression Arabidopsis lines after the high-temperature treatment. Asterisks denote significant differences as determined by a t-test $\left({ }^{* *} \mathrm{P}<0.01\right)$. (c) Relative expression levels of high-temperature resistance-related genes (AtHSP101,AtHSP22.0-ER , AtHSP21, and AtHSP70T-2 ) in WT andMdHSFA9b -overexpression lines at the normal temperature (22degC) and $1 \mathrm{~h}$ after exposure to the high-temperature (45degC) treatment. Each sample was analysed with three biological replicates, each comprising three technical replicates. Asterisks denote significant differences as determined by a t-test $\left({ }^{*} \mathrm{P}<0.05\right)$.

Figure 11. Model of MdNup62 interactions with MdHSFs involved in flowering and heat-stress tolerance in apple.

\section{Supporting Information}

Table S1. Expression of NPC components in different tissues of several apple varieties.

The full names of the different abbreviations are as follows, 'Nagafu No.2' long branches flower buds (FLB), 'Nagafu No.2' short branches flower buds (FSB), 'Nagafu No.2' axillary buds (FYB), 'Qinguan' axillary buds (QYB), 'Nagafu No.2' fruit (FR), 'Yanfu No.3' stem tip (YF3J), 'Yanfu No.6' stem tip (YF6J), and 'M9-T337' root (T337R). Each number after the abbreviation represents a biological repetition.

Table S2. Expression of MdHSFs in different tissues of several apple varieties.

Table S3. MdNup62 yeast double-hybridization screening results.

Table S4. Primers used for qRT-PCR.

Table S5. Primers used for plasmid construction.

Figure S1. Interactions between MdNup62 and MdNup54 in a luciferase (LUC) complementation experiment.

(a) A schematic of the nuclear pore with the cytoplasmic side at the top and the nuclear basket at the bottom for Vertebrate (left) and Malus (right). MdNup62 interacts with MdNup54 forming the central NPC channel.

(b) Interactions between MdNup62 and MdNup54 in a luciferase (LUC) complementation experiment, as well as in our previous study (Zhang et al., 2020). Empty NLUC and empty CLUC, MdNup62-NLUC plus empty CLUC, empty NLUC plus MdNup54-CLUC were used as controls. The LUC complementation experiment was repeated three times, with consistent results.

Figure S2. Genomic PCR analyses of MdNup62 (a),MdHSFA9b (b), and MdHSFA1d (c) in transgenic Arabidopsis lines.

Figure S3. MdHSFA1d promotes flowering in Arabidopsis.

(a) Phenotype of the MdHSFA1d -overexpression Arabidopsis line for flowering time. Bar $=2 \mathrm{~cm}$. (b) Statistical analysis of rosette leaves of Arabidopsis thaliana during bolting. Asterisks denote significant 
differences as determined by a t-test $\left({ }^{*} \mathrm{P}<0.05\right)$. (c) Semi-quantitative RT-PCR analysis of MdHSFA1d expression in Arabidopsis samples. (d) qRT-PCR analysis of MdHSFA1d expression in Arabidopsis samples. Asterisks denote significant differences as determined by a t-test $\left({ }^{*} \mathrm{P}<0.01\right)$. (e) Relative expression levels of flowering genes (AtFT , AtLFY, AtSOC1, andAtAP1) in WT and MdHSFA1d-overexpression lines. Each sample was analysed with three biological replicates, each comprising three technical replicates. Asterisks denote significant differences as determined by a t-test $\left({ }^{*} \mathrm{P}<0.05\right)$.

\section{Figure S4. MdHSFA1d enhanced high-temperature resistance in Arabidopsis.}

(a) Phenotype of the MdHSFA1d -overexpression Arabidopsis line for high-temperature resistance. Bar $=2$ $\mathrm{cm}$. (b) Survival rates of WT andMdHSFA1d -overexpression Arabidopsis lines after the high-temperature treatment. Asterisks denote significant differences as determined by a t-test $(* * \mathrm{P}<0.01)$. (c) Relative expression levels of high-temperature resistance-related genes (AtHSP101,AtHSP22.0-ER , AtHSP21 , and AtHSP70T-2 ) in WT andMdHSFA1d -overexpression lines at the normal temperature (22degC) and $1 \mathrm{~h}$ after exposure to the high-temperature (37degC) treatment. Each sample was analysed with three biological replicates, each comprising three technical replicates. Asterisks denote significant differences as determined by a t-test $(* * \mathrm{P}<0.01)$.

Figure S5. Changes in the levels of accumulated ROS in Arabidopsis leaves under heat-stress conditions . (a) and (b) In situ accumulations of MdHSFA9b (a), MdHSFA1d (b), and superoxide radicals $\left(\mathrm{O}^{2-}\right)$ before (upper panels) and after (lower panels) the heat treatment as revealed by nitro blue tetrazolium staining. Bar $=1 \mathrm{~cm}$.

Reference

Banti V., Mafessoni F., Loreti E., Alpi A. \& Perata P. (2010) The heat-inducible transcription factor HsfA2 enhances anoxia tolerance in Arabidopsis. Plant Physiol , 152, 1471-1483.

Baurle I. \& Dean C. (2006) The Timing of Developmental Transitions in Plants.Cell (Cambridge), 125 , 655-664.

Boeglin M., Fuglsang A.T., Luu D.T., Sentenac H., Gaillard I. \& Cherel I. (2016) Reduced expression of AtNUP62 nucleoporin gene affects auxin response in Arabidopsis. BMC Plant Biol , 16 , 2.

Charng Y., Liu H., Liu N., Chi W., Wang C., Chang S. \& Wang T. (2007) A Heat-Inducible Transcription Factor, HsfA2, Is Required for Extension of Acquired Thermotolerance in Arabidopsis. Plant Physiology ,143, 251-262.

Chen Z., Zhao W., Ge D., Han Y., Ning K., Luo C., Wang S., Liu R., Zhang X. \& Wang Q. (2018) LCM-seq reveals the crucial role ofLsSOC1 in heat-promoted bolting of lettuce (Lactuca sativa L.). The Plant Journal , $95,516-528$.

Cheng Y.T., Germain H., Wiermer M., Bi D., Xu F., Garcia A.V., Wirthmueller L., Despres C., Parker J.E., Zhang Y. \& Li X. (2009) Nuclear Pore Complex Component MOS7/Nup88 Is Required for Innate Immunity and Nuclear Accumulation of Defense Regulators in Arabidopsis. The Plant cell , 21 , 2503-2516.

Cheng Z., Zhang X., Huang P., Huang G., Zhu J., Chen F., Miao Y., Liu L., Fu Y. \& Wang X. (2020) Nup96 and HOS1 Are Mutually Stabilized and Gate CONSTANS Protein Level, Conferring Long-Day Photoperiodic Flowering Regulation in Arabidopsis. The Plant cell , 32, 374-391.

Clough S.J. \& Bent A.F. (1998) Floral dip: a simplified method for Agrobacterium -mediated transformation of Arabidopsis thaliana. The Plant journal : for cell and molecular biology , 16 , 735-743.

Dong C.H., Agarwal M., Zhang Y., Xie Q. \& Zhu J.K. (2006) The negative regulator of plant cold responses, HOS1, is a RING E3 ligase that mediates the ubiquitination and degradation of ICE1. Proceedings of the National Academy of Sciences - PNAS , 103, 8281-8286. 
Dong C.H., Hu X., Tang W., Zheng X., Kim Y.S., Lee B.H. \& Zhu J.K. (2006) A Putative Arabidopsis Nucleoporin, AtNUP160, Is Critical for RNA Export and Required for Plant Tolerance to Cold Stress. Molecular and cellular biology, 26, 9533-9543.

Fan S., Zhang D., Lei C., Chen H., Xing L., Ma J., Zhao C. \& Han M. (2016) Proteome Analyses Using iTRAQ Labeling Reveal Critical Mechanisms in Alternate Bearing Malus prunifolia. Journal of proteome research ,15, 3602-3616.

Gorlich D., Seewald M.J. \& Ribbeck K. (2003) Characterization of Ran-driven cargo transport and the RanGTPase system by kinetic measurements and computer simulation. EMBO J , 22, 1088-1100.

Guitton B., Kelner J., Velasco R., Gardiner S.E., Chagne D. \& Costes E. (2012) Genetic control of biennial bearing in apple. Journal of experimental botany , 63, 131-149.

Hill C.S. (2009) Nucleocytoplasmic shuttling of Smad proteins. Cell Res , 19, 36-46.

Ikeda M., Mitsuda N. \& Ohme-Takagi M. (2011) Arabidopsis HsfB1 and HsfB2b Act as Repressors of the Expression of Heat-Inducible Hsfs But Positively Regulate the Acquired Thermotolerance1[C][W][OA].Plant physiology (Bethesda), 157, 1243-1254.

Ishitani M., Xiong L., Lee H., Stevenson B. \& Zhu J.K. (1998) HOS1, a genetic locus involved in coldresponsive gene expression in arabidopsis.Plant Cell , 10, 1151-1161.

Jacob Y., Mongkolsiriwatana C., Veley K.M., Kim S.Y. \& Michaels S.D. (2007) The Nuclear Pore Protein AtTPR Is Required for RNA Homeostasis, Flowering Time, and Auxin Signaling1[C][W][OA]. Plant physiology (Bethesda) , 144, 1383-1390.

Jung J.H., Park J.H., Lee S., To T.K., Kim J.M., Seki M. \& Park C.M. (2013) The Cold Signaling Attenuator HIGH EXPRESSION OF OSMOTICALLY RESPONSIVE GENE1 Activates FLOWERING LOCUS C Transcription via Chromatin Remodeling under Short-Term Cold Stress in Arabidopsis. The Plant Cell ,25 , 4378-4390.

Komeda Y. (2004) GENETIC REGULATION OF TIME TO FLOWER INARABIDOPSIS THALIANA.Annual Review of Plant Biology , 55 , 521-535.

Kotak S., Port M., Ganguli A., Bicker F. \& von Koskull-Doring P. (2004) Characterization of C-terminal domains ofArabidopsis heat stress transcription factors (Hsfs) and identification of a new signature combination of plant class A Hsfs with AHA and NES motifs essential for activator function and intracellular localization. The Plant Journal , 39, 98-112.

Kotoda N., Iwanami H., Takahashi S. \& Abe K. (2006) Antisense Expression of MdTFL1, a TFL1-like Gene, Reduces the Juvenile Phase in Apple. Journal of the American Society for Horticultural Science, 131, 74-81.

Kotoda N., Wada M., Kusaba S., Kano-Murakami Y., Masuda T. \& Soejima J. (2002) Overexpression of MdMADS5, an APETALA1-like gene of apple, causes early flowering in transgenic Arabidopsis. Plant science (Limerick) ,162, 679-687.

Lazaro A., Mouriz A., Pineiro M. \& Jarillo J.A. (2015) Red Light-Mediated Degradation of CONSTANS by the E3 Ubiquitin Ligase HOS1 Regulates Photoperiodic Flowering in Arabidopsis. The Plant Cell ,27 , 2437-2454.

Li W.M., Tao Y., Yao Y.X., Hao Y.J. \& You C.X. (2010) Ectopic over-expression of two apple Flowering Locus T homologues, MdFT1 and MdFT2, reduces juvenile phase in Arabidopsis. Biologia plantarum ,54, 639-646.

Li Y., Zhang D., An N., Fan S., Zuo X., Zhang X., Zhang L., Gao C., Han M. \& Xing L. (2019) Transcriptomic analysis reveals the regulatory module of apple (Malus $\mathrm{x}$ domestica) floral transition in response to 6-BA.BMC Plant Biol , $19,93$. 
Littlefield O. \& Nelson H.C.M. (1999) A new use for the 'wing' of the 'winged' helix-turn-helix motif in the HSF-DNA cocrystal. Nature structural biology , 6 , 464-470.

Liu D.D., Sun X.S., Liu L., Shi H.D., Chen S.Y. \& Zhao D.K. (2019) Overexpression of the Melatonin Synthesis-Related Gene SlCOMT1 Improves the Resistance of Tomato to Salt Stress. Molecules, 24.

Liu J., Feng L., Gu X., Deng X., Qiu Q., Li Q., Zhang Y., Wang M., Deng Y., Wang E., He Y., Baurle I., Li J., Cao X. \& He Z. (2019) An H3K27me3 demethylase-HSFA2 regulatory loop orchestrates transgenerational thermomemory in Arabidopsis. Cell research , 29 , 379-390.

Liu K., Feng S., Pan Y., Zhong J., Chen Y., Yuan C. \& Li H. (2016) Transcriptome Analysis and Identification of Genes Associated with Floral Transition and Flower Development in Sugar Apple (Annona squamosa L.). Front Plant Sci, $7,1695$.

Liu L., Guo L., Li M.H., Fu W.D. \& Luan Q. (2020) Changes of chilling and heat accumulation of apple and their effects on the first flowering date in the main planting areas of northern China. Chinese Journal of Applied Ecology , 31, 2457-2463.

Livak K.J. \& Schmittgen T.D. (2001) Analysis of Relative Gene Expression Data Using Real-Time Quantitative PCR and the 2- $\Delta \Delta$ CT Method. Methods ,25, 402-408.

Luo Y., Wang Z., Ji H., Fang H., Wang S., Tian L. \& Li X. (2013) An Arabidopsis homolog of importin beta1 is required for ABA response and drought tolerance. Plant $J$, 75 , 377-389.

Mishra S.K. (2002) In the complex family of heat stress transcription factors, HsfA1 has a unique role as master regulator of thermotolerance in tomato. Genes $\&$ development, 16 , 1555-1567.

Nishizawa-Yokoi A., Nosaka R., Hayashi H., Tainaka H., Maruta T., Tamoi M., Ikeda M., Ohme-Takagi M., Yoshimura K., Yabuta Y. \& Shigeoka S. (2011) HsfA1d and HsfA1e involved in the transcriptional regulation of HsfA2 function as key regulators for the Hsf signaling network in response to environmental stress. Plant Cell Physiol , 52, 933-945.

Nover L., Bharti K., Doring P., Mishra S.K., Ganguli A. \& Scharf K.D. (2001) Arabidopsis and the heat stress transcription factor world: how many heat stress transcription factors do we need? Cell Stress Chaperones , 6 , 177-189.

Parry G. (2013) Assessing the function of the plant nuclear pore complex and the search for specificity. Journal of Experimental Botany .

Parry G. (2014) Components of the Arabidopsis nuclear pore complex play multiple diverse roles in control of plant growth. Journal of experimental botany , 65, 6057-6067.

Parry G., Ward S., Cernac A., Dharmasiri S. \& Estelle M. (2006) The Arabidopsis SUPPRESSOR OF AUXIN RESISTANCE proteins are nucleoporins with an important role in hormone signaling and development. Plant Cell , $18,1590-1603$.

Qian J., Chen J., Liu Y.F., Yang L.L., Li W.P. \& Zhang L.M. (2014) Overexpression of Arabidopsis HsfA1a enhances diverse stress tolerance by promoting stress-induced Hsp expression. Genetics and molecular research , 13, 1233-1243.

Robles L.M., Deslauriers S.D., Alvarez A.A. \& Larsen P.B. (2012) A loss-of-function mutation in the nucleoporin AtNUP160 indicates that normal auxin signalling is required for a proper ethylene response in Arabidopsis. Journal of experimental botany , 63, 2231-2241.

Romanovskaja D. \& Bakšiene E. (2009) Influence of climatic warming on beginning of flowering of apple tree (Malus domestica Barkh.) in Lithuania. , 2009, 7 (1), 87-96. Agronomy Research .

Roth C. \& Wiermer M. (2012) Nucleoporins Nup160 and Seh1 are required for disease resistance in Arabidopsis. Plant Signal Behav ,7 , 1212-1214. 
Scharfa K., Berberich T., Ebersberger I. \& Nover L. (2012) The plant heat stress transcription factor (Hsf) family Structure, function, and evolution. Biochimica et Biophysica Acta .

Schramm F., Larkindale J., Kiehlmann E., Ganguli A., Englich G., Vierling E. \& Von Koskull-Döring P. (2008) A cascade of transcription factor DREB2A and heat stress transcription factor HsfA3 regulates the heat stress response of Arabidopsis. The Plant Journal , 53 , 264-274.

Takizawa C.G., Weis K. \& Morgan D.O. (1999) Ran-independent nuclear import of cyclin B1-Cdc2 by importin beta. Proc Natl Acad Sci U S A ,96 , 7938-7943.

Tamura K., Fukao Y., Iwamoto M., Haraguchi T. \& Hara-Nishimura I. (2010) Identification and characterization of nuclear pore complex components in Arabidopsis thaliana. Plant Cell , 22 , 4084-4097.

Teotia S. \& Tang G. (2015) To Bloom or Not to Bloom: Role of MicroRNAs in Plant Flowering. Molecular Plant , 8, 359-377.

Tian X., Wang F., Zhao Y., Lan T., Yu K., Zhang L., Qin Z., Hu Z., Yao Y., Ni Z., Sun Q., Rossi V., Peng H. \& Xin M. (2020) Heat shock transcription factor A1b regulates heat tolerance in wheat and Arabidopsis throughOPR 3 and jasmonate signalling pathway. Plant Biotechnology Journal ,18 , 1109-1111.

Trankner C., Lehmann S., Hoenicka H., Hanke M.V., Fladung M., Lenhardt D., Dunemann F., Gau A., Schlangen K., Malnoy M. \& Flachowsky H. (2010) Over-expression of an FT-homologous gene of apple induces early flowering in annual and perennial plants. Planta, 232, 1309-1324.

Wada M., Cao Q.F., Kotoda N., Soejima J. \& Masuda T. (2002) Apple has two orthologues of FLORICAULA/LEAFY involved in flowering. Plant Mol Biol , 49, 567-577.

Wang N., Liu W., Yu L., Guo Z., Chen Z., Jiang S., Xu H., Fang H., Wang Y., Zhang Z. \& Chen X. (2020) HEAT SHOCK FACTOR A8a Modulates Flavonoid Synthesis and Drought Tolerance. Plant Physiol , 184 , 1273-1290.

Wiermer M., Cheng Y.T., Imkampe J., Li M., Wang D., Lipka V. \& Li X. (2012) Putative members of the Arabidopsis Nup107-160 nuclear pore sub-complex contribute to pathogen defense. Plant J , 70, 796-808.

Xiang J., Ran J., Zou J., Zhou X., Liu A., Zhang X., Peng Y., Tang N., Luo G. \& Chen X. (2013) Heat shock factor OsHsfB2b negatively regulates drought and salt tolerance in rice. Plant Cell Rep , 32, 1795-1806.

Xing L., Zhang D., Li Y., Shen Y., Zhao C., Ma J., An N. \& Han M. (2015) Transcription Profiles Reveal Sugar and Hormone Signaling Pathways Mediating Flower Induction in Apple (Malus domestica Borkh.).Plant and cell physiology , $5 \mathbf{6}, 2052-2068$.

Xu X.M. \& Meier I. (2008) The nuclear pore comes to the fore. Trends in Plant Science, 13 , 20-27.

Xu X.M., Rose A., Muthuswamy S., Jeong S.Y., Venkatakrishnan S., Zhao Q. \& Meier I. (2007) NUCLEAR PORE ANCHOR, the Arabidopsis homolog of Tpr/Mlp1/Mlp2/megator, is involved in mRNA export and SUMO homeostasis and affects diverse aspects of plant development. Plant Cell ,19 , 1537-1548.

Yang Y., Wang W., Chu Z., Zhu J. \& Zhang H. (2017) Roles of Nuclear Pores and Nucleo-cytoplasmic Trafficking in Plant Stress Responses.Frontiers in plant science , 8, 574.

Yao F., Song C., Wang H., Song S., Jiao J., Wang M. \& Zheng X. (2020) Genome-Wide Characterization of the HSP20 Gene Family Identifies Potential Members Involved in Temperature Stress Response in Apple.Frontiers in genetics , 11,609184.

Zhang A., Wang S., Kim J., Yan J., Yan X., Pang Q. \& Hua J. (2020) Nuclear pore complex components have temperature-influenced roles in plant growth and immunity. Plant Cell Environ, 43, 1452-1466.

Zhang C., An N., Jia P., Zhang W., Liang J., Zhang X., Zhou H., Ma W., Han M., Xing L. \& Ren X. (2020) Genomic identification and expression analysis of nuclear pore proteins in Malus domestica. Scientific reports ,10, 17426 . 
Zhang S., Gottschalk C. \& van Nocker S. (2019) Genetic mechanisms in the repression of flowering by gibberellins in apple (Malus x domestica Borkh.). BMC Genomics, 20 , 747.

Zhang Y. \& Li X. (2005) A Putative Nucleoporin 96 Is Required for Both Basal Defense and Constitutive Resistance Responses Mediated bysuppressor of npr1-1 ,constitutive 1. The Plant Cell , 17 , 1306-1316.

ZHOU B., SUN J., LIU S., JIN W., ZHANG Q. \& WEI Q. (2016) Dwarfing apple rootstock responses to elevated temperatures: A study on plant physiological features and transcription level of related genes. Journal of Integrative Agriculture, 15, 1025-1033.

Zhu Y., Wang B., Tang K., Hsu C.C., Xie S., Du H, Yang Y., Tao W.A. \& Zhu J.K. (2017) An Arabidopsis Nucleoporin NUP85 modulates plant responses to ABA and salt stress. PLoS Genet, 13, e1007124.

Zinsmeister J., Berriri S., Basso D.P., Ly Vu B., Dang T.T., Lalanne D., Da Silva E.A.A., Leprince O. \& Buitink J. (2020) The seed-specific heat shock factorA9 regulates the depth of dormancy inMedicago truncatula seeds viaABA signalling. Plant, Cell \& Environment, 43 , 2508-2522.

\section{Hosted file}

Figures_20201229.pdf available at https://authorea.com/users/389962/articles/504444-mdnup62interactions-with-mdhsfs-involved-in-flowering-and-heat-stress-tolerance-in-apple 